OPEN ACCESS

Edited by:

Sergej Ostojic,

University of Novi Sad, Serbia

Reviewed by:

Zhaowei Kong,

University of Macau, Macau José Ramón Alvero Cruz,

University of Malaga, Spain

*Correspondence:

Emma Roca

info@projectesummit.com

Francesc Villarroya

fvillarroya@ub.edu

${ }^{\dagger}$ These authors have contributed equally to this work

Specialty section:

This article was submitted to Exercise Physiology,

a section of the journal

Frontiers in Physiology

Received: 08 April 2020

Accepted: 30 October 2020

Published: 19 November 2020

Citation:

Campderrós $L$

Sánchez-Infantes $D$, Villarroya J, Nescolarde L, Bayès-Genis A Cereijo $R$, Roca $E$ and Villarroya $F$ (2020) Altered GDF15 and FGF21 Levels in Response to Strenuous

Exercise: A Study in Marathon

Runners. Front. Physiol. 11:550102. doi: 10.3389/fphys.2020.550102

\section{Altered GDF15 and FGF21 Levels in Response to Strenuous Exercise: A Study in Marathon Runners}

\author{
Laura Campderrós ${ }^{1,2+}$, David Sánchez-Infantes ${ }^{2,3 \dagger}$, Joan Villarroya ${ }^{1,2}$, Lexa Nescolarde ${ }^{4,5}$, \\ Antoni Bayès-Genis ${ }^{6,7,8}$, Rubén Cereijo ${ }^{1,2}$, Emma Roca ${ }^{4 *}$ and Francesc Villarroya ${ }^{1,2 *}$ \\ 'Departament de Bioquimica i Biomedicina Molecular, University of Barcelona, Barcelona, Spain, ${ }^{2}$ CIBER Fisiopatologia \\ de la Obesidad y Nutrición, Madrid, Spain, ${ }^{3}$ Institut de Recerca Germans Trias i Pujol, Barcelona, Spain, ${ }^{4}$ Research Centre \\ for Biomedical Engineering, Universitat Politècnica de Catalunya, Barcelona, Spain, ${ }^{5}$ Department of Electronic Engineering, \\ Universitat Politècnica de Catalunya, Barcelona, Spain, ${ }^{6}$ Hospital Universitari Germans Trias i Pujol, Barcelona, Spain, \\ ${ }^{7}$ Department of Medicine, Universitat Autònoma de Barcelona, Barcelona, Spain, ${ }^{8}$ CIBER de Enfermedades \\ Cardiovasculares, Madrid, Spain
}

Background: Recreational marathon runners face strong physiological challenges. Assessment of potential biomarkers for the biological responses of runners will help to discriminate individual race responsiveness and their physiological consequences. This study sought to analyze the changes in the plasma levels of GDF15 and FGF21, novel endocrine factors related to metabolic stress, in runners following the strenuous exercise of a marathon race.

Methods: Blood samples were obtained from eighteen male runners (mean $\pm S D$, age: $41.7 \pm 5.0$ years, BMl: $23.6 \pm 1.8) 48 \mathrm{~h}$ before, immediately after, and $48 \mathrm{~h}$ after a marathon race, and from age-matched sedentary individuals. The level of GDF15, FGF21, and 38 additional biochemical and hematological parameters were determined.

Results: The basal levels of GDF15 and FGF21 did not differ between runners before the race and sedentary individuals. Significant increases in the mean levels of GDF15 (4.2-fold) and FGF21 (20-fold) were found in runners immediately after the race. The magnitudes of these increases differed markedly among individuals and did not correlate with each other. The GDF15 and FGF21 levels had returned to the basal level $48 \mathrm{~h}$ postrace. The post-race value of GDF15 (but not FGF21) correlated positively with increased total white cell count $(r=0.50, P=0.01)$ and neutrophilia $(r=0.10, P=0.01)$.

Conclusion: GDF15 and FGF21 are transiently increased in runners following a marathon race. The induction of GDF15 levels is associated with alterations in circulating immune cells levels.

Keywords: strenuous exercise, GDF15, FGF21, marathon athletes, biomarker

\section{INTRODUCTION}

Recreational running is a widespread activity and the marathon (a 42.2- $\mathrm{km}$ running race named for the Greek legend) is growing in popularity, with modern-day marathons drawing thousands of contestants. Recreational marathon runners face strong physiological challenges and multiple studies have reported alterations in physiological parameters and associated blood parameters 
during and after a marathon race (Kratz et al., 2002; Reid et al., 2004; Kobayashi et al., 2005; Bird et al., 2014). The commonly reported findings include increased levels of biomarkers for exertional myolysis as well as increased neutrophilia and monocytosis (Kratz et al., 2002; Bird et al., 2014). Additional biomarkers are needed to help discriminate differential individual race responsiveness.

Growth/differentiation factor-15 (GDF15), which is also called macrophage inhibitor (MIC-1) and non-steroidal antiInflammatory drug-activated gene-1 (NAG-1), is a member of the TGF $\beta$ superfamily. Although the functional role of GDF15 is not fully known, elevated GDF15 levels in blood have been reported as a biomarker of several pathological conditions, such as cancer, inflammatory disorders, cardiovascular disease and type 2 diabetes, and as a biomarker of mortality of any kind (Adela and Banerjee, 2015; Hagström et al., 2017; Tsai et al., 2018). The lack of an identified GDF15 receptor previously hampered efforts to assess the physiological role of GDF15 but recent work identified a receptor for GDF15 (GFRAL) in brain, and showed that it accounts for the anorexigenic actions of GDF15 (Emmerson et al., 2017; Hsu et al., 2017; Mullican et al., 2017; Yang et al., 2017). GDF15 has been reported to exert antiinflammatory actions of GDF15 in peripheral cell systems, such as eosinophils and macrophages (Bootcov et al., 1997; Artz et al., 2016), via yet-unidentified receptor systems.

Elevated GDF15 levels have been reported as a biomarker of neuromuscular diseases of genetic origin caused by alterations in the mitochondrial genome (Fujita et al., 2015; Yatsuga et al., 2015). Indeed, experimentally induced alterations in muscle cell mitochondrial bioenergetics reportedly trigger enhancements in GDF15 gene expression and muscle cell release (Montero et al., 2016). In this sense, the secretion of GDF15 in muscle parallels that of fibroblast growth factor-21 (FGF21), which was recently proposed to be a biomarker of diseases caused by mitochondrial DNA mutations (Suomalainen et al., 2011; Ribas et al., 2014). In children affected by distinct mitochondrial diseases, the levels of GDF15 and FGF21 levels are increased and highly correlated with one another (Montero et al., 2016).

Numerous studies have reported that FGF21 levels increase in response to experimental acute exercise (Cuevas-Ramos et al., 2012; Kim et al., 2013; Hansen et al., 2015, 2016; Slusher et al., 2015; Tanimura et al., 2016; Morville et al., 2018; Sargeant et al., 2018). GDF15 has also been reported to exhibit transient elevations following an experimental single bout of exercise (Kleinert et al., 2018), a cycling race (Conte et al., 2020) and relatively time-limited sports activities such as a soccer match (Sanchis-Gomar et al., 2013) or a training session of rugby players (Galliera et al., 2014). Increased levels of GDF15 in athletes immediately after a $247-\mathrm{km}$ race (the so-called Spartathlon) has also been reported (Tchou et al., 2009). However, the literature lacks a thorough characterization of changes in GDF15 and their comparison with FGF21 following a marathon race, which is the most commonly practiced recreational race involving strenuous exercise.

Given the potential roles of FGF21 and GDF15 in the physiological response to exercise, we wanted to explore whether these factors could contribute to the assessment of the physiological response to a marathon race. Here, we determined the levels of GDF15 and FGF21 in marathon runners before race (basal levels), immediately after the race (acute response to strenuous exercise) and 2 days after the race (short time recovery). We also sought to correlate these data with standard parameters previously reported to be altered in athletes after a marathon race (Kratz et al., 2002; Reid et al., 2004; Kobayashi et al., 2005; Bird et al., 2014).

\section{METHODS}

The study was designed to analyze the changes of GDF15 and FGF21 levels in recreational athletes before and after a marathon race held at Barcelona on March 17, 2017.

Volunteers were recruited as part of the "SUMMIT project" ("Health in Ultra-Marathon and their Limits"), which sought to evaluate the behavior of certain clinical parameters among runners competing in different races. The SUMMIT project was approved by an institutional review board (IIBSP-SUMMIT2016-2) and all participants provided written informed consent to the current study. The study sample included 18 recreational male athletes (mean \pm SD age: $41.7 \pm 5.0$ years, BMI: $23.6 \pm 1.8$ ). Sample size was dependent on availability of volunteers and was not based on power calculations; however, sample size was in the range of that resulting from power calculations in studies of individuals at rest and after a single bout of exercise (Yatsuga et al., 2015; Poulsen et al., 2020). The median (interquartile range, IQR) years of training was 7 (5-11) years and the median (IQR) of weekly training hours was $6(5-8) \mathrm{h} /$ week. The median (IQR) race time (h:min:s) was 3:32:44 (3:18:50-3:51:46).

The participants were provided with guidelines to maintain adequate levels of hydration during the race. The first liquid intake was programmed at $60 \mathrm{~min}$ of the race; the dosage consisted of $400 \mathrm{ml}$ for lighter/slower runners and $800 \mathrm{ml}$ for heavier/faster runners, and runners were asked to drink $100-150 \mathrm{ml}$ every $15-20 \mathrm{~min}$. Commercialized beverages were provided to participants; the drinks averaged $480 \mathrm{mg} / \mathrm{L}$ for $\mathrm{Na}^{+}$, $85 \mathrm{mg} / \mathrm{L}$ for $\mathrm{K}^{+}$, and $45 \mathrm{mg} / \mathrm{L}$ for $\mathrm{Mg}^{+}$.

Three 10-mL blood samples were obtained from the antecubital vein in EDTA vacutainers at $48 \mathrm{~h}$ before the marathon (baseline), at completion (within the $10 \mathrm{~min}$ of an individual completing the race and before they drank any fluid or emptied the bladder) and $48 \mathrm{~h}$ after the race. Blood samples were centrifuged at $3,000 \mathrm{rpm}$ at $4^{\circ} \mathrm{C}$ for $10 \mathrm{~min}$ in a bench-top centrifuge. Serum samples were aliquoted and stored on dry ice, and all samples were frozen at $-80^{\circ} \mathrm{C}$.

Biochemical and hematological parameters were determined in blood at the Clinical Biochemistry and Hematology facilities at Hospital Germans Trias i Pujol (Badalona, Spain). Blood glucose lipids, urea, total protein, ions, alanine aminotransferase (ALT), aspartate aminotransferase (AST), lactate dehydrogenase (LDH), and gamma-glutamyl transpeptidase (GGT) were analyzed by routine clinical chemistry and using an AU-5800 Chemistry Analyzer (Beckman Coulter Inc., Brea, CA, United States). Complete blood counts were obtained using a Unicel DxH800 automated hematology analyzer (Beckman Coulter, Miami, 
FL, United States). Troponin $\mathrm{T}$ was measured from serum, using a Highly Sensitive Troponin-T assay on a Cobas e601 platform (Roche Diagnostics, Barcelona, Spain). The interassays coefficients of variation (CV) in the analytical assays are shown in Supplementary Table 1. FGF21 levels (intraassay CV 2.0\%, inter-assay CV 3.3\%) and GDF15 levels (intraassay CV 2.6\%, inter-assay CV 5.3\%) were measured using human-specific ELISA kits (R\&D Systems, United Kingdom, and Biovendor, Czechia, respectively). For comparison purposes, an age-matched and BMI-matched group of healthy male volunteers, with a sedentary behavioral profile (no recreational or relevant occupational-related exercise activity), was recruited among personnel of the University of Barcelona $(N=19$, mean \pm SD age: $41.4 \pm 3.0$ years) and studied.

The normality of distribution of the variables was checked by the Shapiro-Wilk test and the homogeneity of variances was assessed by Levene's test. Repeated-measures ANOVA test was used to determine the effect of the marathon on variables measured at $24 \mathrm{~h}$ pre-race, immediately post-race and $48 \mathrm{~h}$ postrace using the multiple-comparison Bonferroni tests. Pearson correlation coefficient was applied according to the normal distribution of variables. The level of statistical significance was set at $P<0.05$. IBM $^{\circledR}$ SPSS $^{\circledR}$ version 24.0 (Armonk, NY, United States) was used for data analysis.

\section{RESULTS}

Immediately after the race, the participating runners showed an increase in their total white blood cell (WBC) count; this was associated with increased numbers of neutrophils, monocytes, and basophils but reduced numbers of lymphocytes and eosinophils (Table 1). These changes were totally reversed at $48 \mathrm{~h}$ after the race, when the values for these parameters were not statistically different from the basal values. Significant increases were also observed in the levels of urea, creatinine, calcium, total protein, sodium, potassium, and bilirubin immediately after the race; all of these parameters also returned to basal levels with the exception of potassium, which remained high at $48 \mathrm{~h}$ postrace, and total protein which was decreased below the basal level at $48 \mathrm{~h}$ post-race. The magnesium and phosphorus levels were transiently reduced after the race and normalized totally (magnesium) or partially (phosphorus) $48 \mathrm{~h}$ later. The levels of $\mathrm{LDH}$ and troponin were significantly induced immediately after the race; at $48 \mathrm{~h}$ after the race, troponin had returned to the basal level whereas $\mathrm{LDH}$ remained significantly elevated. The creatine kinase level was significantly higher immediately after the race compared to basal level and was further increased at $48 \mathrm{~h}$ postrace. Finally, the levels of ALT and C-reactive protein levels were increased relative to their basal levels only at $48 \mathrm{~h}$ post-race.

The basal levels of plasma GDF15 and FGF21 did not significantly differ between pre-race athletes and sedentary individuals (Figure 1A), indicating that chronic training does not influence the basal levels of these two bioactive factors. Immediately after the race, the levels of both GDF15 and FGF21 levels were dramatically increased in athletes; there was a 4.2 -fold increase in the mean GDF15 level and a $~ 20$-fold increase in the mean FGF21 level (Figure 1A), and the extents of these increases differed markedly among the studied individuals (Figure 1B). The plasma levels of GDF15 and FGF21 had returned to nearbasal levels at $48 \mathrm{~h}$ post-race. There was no significant correlation in the basal levels of GDF15 and FGF21 or the extent of their increases immediately after the race (Table 2). There was also no significant correlation between the increase of GDF15 or FGF21 and the time spent in the race or any other race-related variable. Among the 32 distinct physiological and blood biochemical and hematological parameters tested herein, the basal (prerace) level of GDF15 was positively correlated with that of ALT (Supplementary Table 2) and the GDF15 level immediately after the race was positively correlated with the WBC count, neutrophil count, urea level, and ALT level (Table 2). The basal levels of FGF21 correlated positively with the WBC count, platelet count, monocyte count, total protein level, and C-reactive protein level (Supplementary Table 2), and correlated negatively with the urea level. The peak levels of FGF21 observed just after the race correlated positively with glycemia and the total protein levels, with marginal statistically significance (Table 2 ).

\section{DISCUSSION}

Overall, the alterations in blood parameters observed in the current study are largely concordant with previous findings on the physiological response to marathon running. We found increases in the levels of total protein, urea, creatinine, bilirubin, creatine kinase, $\mathrm{LDH}$, and troponin after the race; these findings paralleled those of a previous study in marathon runners (Bird et al., 2014) and were consistent with a scenario of exertional muscle injury and hemolysis. Some of these parameters returned to their basal values by $48 \mathrm{~h}$ post-race, whereas others (e.g., creatine kinase, $\mathrm{LDH}$, and troponin) did not; again, this was consistent with previous reports (Kobayashi et al., 2005; Bird et al., 2014). We also confirmed that the increase in total WBC count was mainly due to increased numbers of neutrophils and monocytes (Kratz et al., 2002; Reid et al., 2004), which is commonly attributed to an inflammatory reaction to exertionrelated tissue injury.

Regarding our novel results, we herein report that the plasma levels of GDF15 and FGF21 were both increased immediately after the marathon race and returned to normal levels within $48 \mathrm{~h}$ post-race. The extent of these transient bursts in GDF15 and FGF21 concentration was very variable among individuals, was not associated with any of the studied intrinsic exerciseassociated traits (i.e., extent of prior training, duration of the race, etc.), and the degrees to which GDF15 and FGF21 increased were not correlated with each other in studied runners.

This is one of only a few studies to focus on how exercise affect the level of GDF15. Studies performed in soccer players after a match and rugby players after training reported a significant increase in GDF15 levels in blood (Sanchis-Gomar et al., 2013; Galliera et al., 2014), and a recent experimental study showed that there was a significant rise in GDF15 levels among volunteers who exercised at $67 \%$ of their VO2max for $1 \mathrm{~h}$ (Kleinert et al., 2018). In these reports, the increase in GDF15 was around 1.5- to 
2 -fold, which is much less than the more than fourfold increase found here after marathon race-associated strenuous exercise. Only a study in spartathlon runner found a rise in GDF15 levels in the fourfold range found here for marathon runners (Tchou et al., 2009) whereas around threefold increase in GDF51 levels were reported after a long distance cycling race (Conte et al., 2020). During the editorial processing of the current article, a study in Marathon runners in Poland has been reported (Kaleta-Duss et al., 2020) showing a rise in GDF15 levels immediately after the race and normalization thereafter, confirming our findings. In that study, focused to cardiovascular markers, a significant increase in the biomarkers of altered cardiac function BNP, NT-proANP, H-FABP, and Gal-3 immediately after the race was also found; however, they did not correlate with GDF15.
Although the rise in GDF15 may be related to changes in cardiac hemodynamic volume and pressure overload immediately after the marathon, it has been proposed that GDF15 does not appear as reliable to track specifically cardiorespiratory fitness in acute exercise, possibly because of the contribution of other noncardiac processes to the GDF15 rise in exercise (Kaleta-Duss et al., 2020). Nonetheless, some authors found GDF15 levels to be associated with impairment in exercise capacity in patients with the heart failure syndrome (Stahrenberg et al., 2010) whereas others do not consider GDF15 as a reliable biomarker of exercise capacity in heart failure patients (Fudim et al., 2020).

Concerning FGF21, the previous studies reported -twofold to fourfold increases in FGF21 after experimental bouts of acute exercise (1-3 h) (Kim et al., 2013; Hansen et al., 2015, 2016;

TABLE 1 | Circulating parameters measured before (pre-marathon), immediately after (post-marathon) and $48 \mathrm{~h}$ after the marathon race.

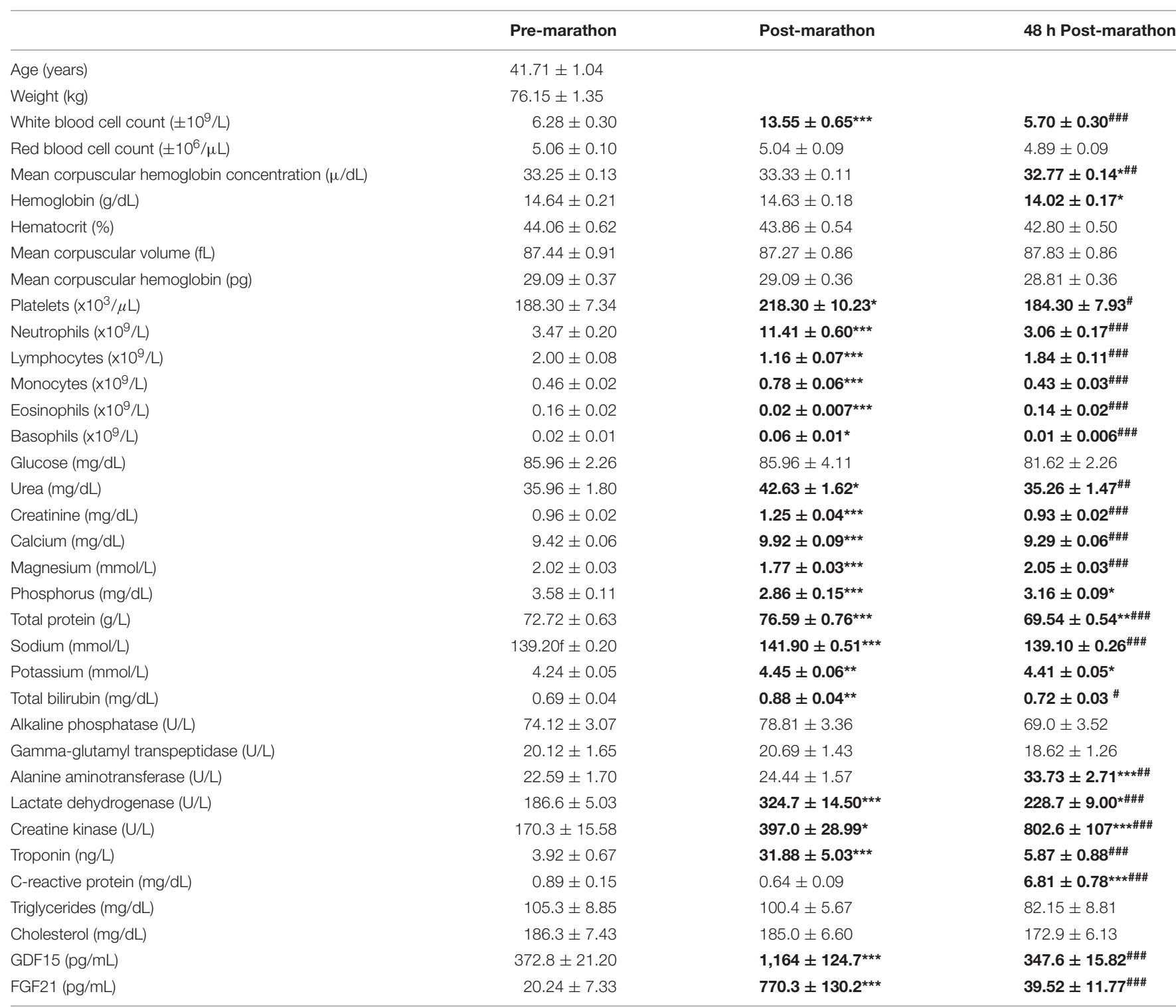

${ }^{*} P<0.05,{ }^{* *} P<0.01$, and ${ }^{* * *} P<0.001$ relative to the measurements before the race and ${ }^{\#} P<0.05$, $\# \#<0.01$, and ${ }^{\# \# \#} P<0.001$ relative to the measurements immediately after the race. Bold lettering is shown when $P<0.05$. 
A

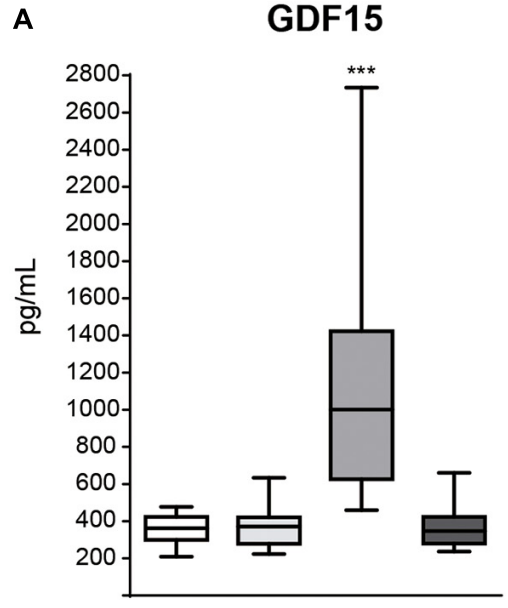

B

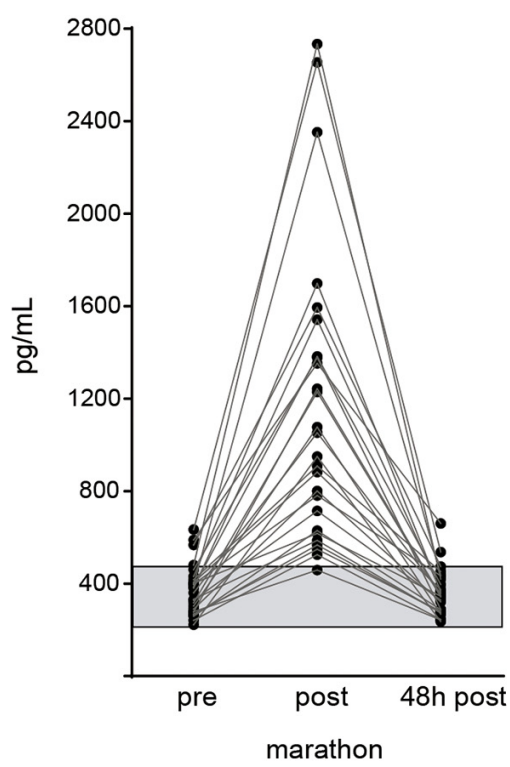

FGF21

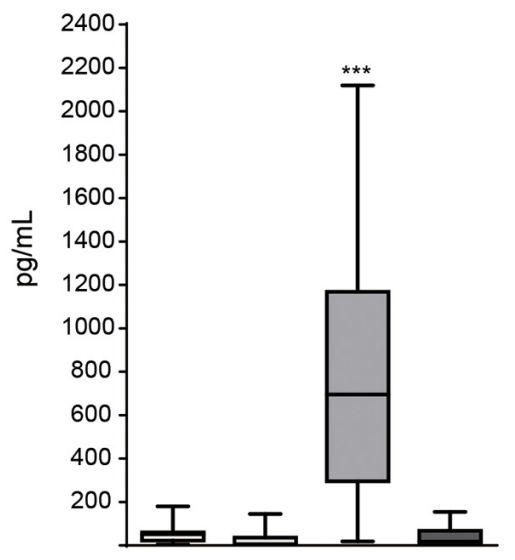

FGF21

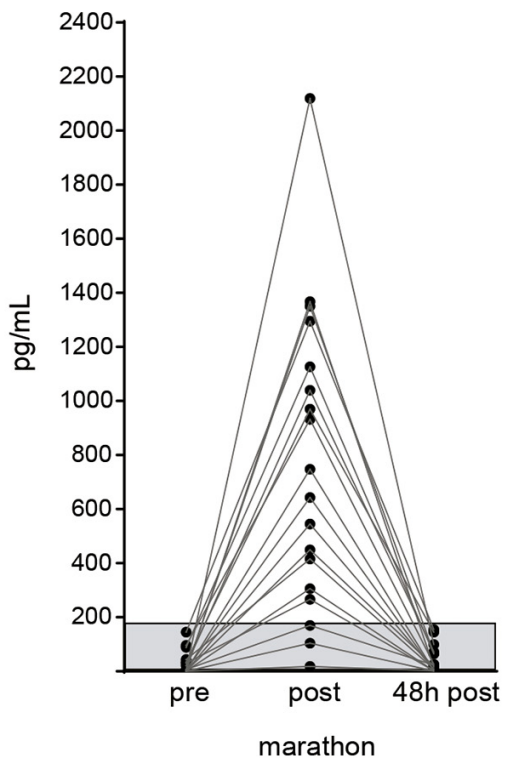

FIGURE 1 | Levels of GDF15 and FGF21 in marathon runners $48 \mathrm{~h}$ before the race (pre-marathon), immediately after the race (post-marathon) and $48 \mathrm{~h}$ after the race, and comparison with levels in a sedentary control population. (A) Box and whisker plot representation of GDF15 and FGF21 levels, ${ }^{* \star *} P<0.001$ relative to pre-marathon values. (B) Individual changes in the levels of GDF15 and FGF21 in the three time points analyzed. Gray bar corresponds to means \pm SEM values in the sedentary control population.

Slusher et al., 2015; Morville et al., 2018; Sargeant et al., 2018), whereas we observed a much greater increase of around 20 -fold in the level of FGF21 immediately after the runners completed the marathon race.

It is worth mentioning that, beyond exercise, the blood levels of GDF15 and FGF21 are also increased in pathological contexts, such as in patients affected by neuromuscular diseases caused by mitochondria DNA mutations (Suomalainen et al., 2011). Moreover, GDF15 and FGF21 were reported to be actively released by human muscle cells after experimental mitochondrial insults in vitro (Ribas et al., 2014; Montero et al., 2016). However, not all the above mentioned studies found correlations between the high levels of GDF15 and FGF21 in patients, and it has been proposed that GDF15 may have value as a biomarker of muscle damage, whereas FGF21 may be more strongly associated with mitochondrial-specific pathologies (Lehtonen et al., 2016).

The lack of correlation between the bursts of the two molecules reported here suggests that distinct physiological processes (and possibly tissues of origin) are involved in these increases. Given that the increase in FGF21 is associated with decreased glycemia and the liver is the main site of FGF21 release under physiological conditions, it is likely that the liver was the main source of the FGF21 increase seen immediately after the marathon race. 
This has been proposed for other exercise-induced changes in FGF21 levels and it is consistently with the behavior of other hepatokines (Weigert et al., 2019). For GDF15, a recent study indicated that the rise in GDF15 after a single bout of exercise is associated with increased GDF15 gene expression in skeletal muscle (Laurens et al., 2020). From this, we hypothesize that muscle may be the source of the high-level induction of GDF15 seen immediately after the marathon race, although correlation with ALT levels does not allow to rule out a potential role of hepatic stress contributing to high GDF15 levels. Further studies are clearly required in order to identify the tissue sources that lead to the rise in GDF15 and FGF21 levels in marathon runners. This may be relevant in order to identify critical sites of tissue stress due to the race indistinct individuals according to their rise in the GDF15 and/or FGF21 biomarkers.
Our study has several limitations. The sample is relatively small and limited to recreational runners, and further data on the characteristics of individuals beyond biochemical and hematological data (e.g., body composition, performance level, pacing, and internal load) could have strengthened the study. The correlative nature of some of our findings is also an obvious limitation. However, our data allow us to conclude that: (a) both GDF15 and FGF21 are dramatically induced immediately after the strenuous exercise associated with a marathon race; (b) the extents to which GDF15 and FGF21 are induced are highly variable among runners and do not correlate with each other; (c) both parameters recover to baseline within 2 days postrace; and (d) the rises in GDF15 and FGF21 immediately after the race are distinctly correlated with alterations in biochemical and hematological biomarkers of the physiological response

TABLE 2 | Linear relationship of the post-marathon GDF15 levels and FGF21 levels with the post-marathon levels of circulating parameters.

\begin{tabular}{|c|c|c|c|c|}
\hline & \multicolumn{2}{|c|}{ GDF15 } & \multicolumn{2}{|c|}{ FGF21 } \\
\hline & $r$ & $\boldsymbol{P}$ & $r$ & $P$ \\
\hline GDF15 & - & - & 0.394 & 0.106 \\
\hline FGF21 & 0.394 & 0.106 & - & - \\
\hline White blood cell count $\left(\times 10^{9} / \mathrm{L}\right)$ & 0.455 & $0.022^{*}$ & 0.930 & 0.237 \\
\hline Red blood cell count $\left(\times 10^{6} / \mu \mathrm{L}\right)$ & -0.067 & 0.751 & -0.237 & 0.343 \\
\hline Mean corpuscular hemoglobin concentration (g/dL) & -0.129 & 0.539 & 0.061 & 0.811 \\
\hline Hemoglobin (g/dL) & -0.054 & 0.799 & -0.202 & 0.422 \\
\hline Hematocrit (\%) & -0.015 & 0.942 & -0.217 & 0.387 \\
\hline Mean corpuscular volume (fL) & 0.113 & 0.591 & 0.149 & 0.555 \\
\hline Mean corpuscular hemoglobin (pg) & 0.058 & 0.782 & 0.134 & 0.596 \\
\hline Platelets $\left(\times 10^{3} / \mu \mathrm{L}\right)$ & 0.084 & 0.689 & 0.374 & 0.126 \\
\hline Neutrophils $\left(\times 10^{9} / \mathrm{L}\right)$ & 0.506 & $0.010^{\star \star}$ & 0.314 & 0.205 \\
\hline Lymphocytes (×109/L) & -0.225 & 0.279 & -0.059 & 0.816 \\
\hline Monocytes $\left(\times 10^{9} / \mathrm{L}\right)$ & 0.326 & 0.112 & 0.319 & 0.198 \\
\hline Eosinophils (×109/L) & -0.368 & 0.071 & -0.152 & 0.547 \\
\hline Basophils $\left(\times 10^{9} / \mathrm{L}\right)$ & 0.241 & 0.247 & 0.050 & 0.843 \\
\hline Glucose (mg/dL) & -0.136 & 0.518 & -0.470 & $0.049^{\star}$ \\
\hline Urea (mg/dL) & 0.661 & $0.0003^{\star \star \star}$ & 0.004 & 0.989 \\
\hline Creatinine (mg/dL) & 0.349 & 0.087 & 0.103 & 0.684 \\
\hline Calcium (mg/dL) & 0.122 & 0.560 & 0.155 & 0.539 \\
\hline Magnesium (mmol/L) & 0.062 & 0.770 & -0.038 & 0.881 \\
\hline Phosphorus (mg/dL) & -0.129 & 0.541 & -0.461 & 0.055 \\
\hline Total protein (g/L) & 0.140 & 0.504 & 0.479 & $0.044^{\star}$ \\
\hline Sodium (mmol/L) & -0.166 & 0.429 & -0.069 & 0.786 \\
\hline Potassium (mmol/L) & 0.373 & 0.066 & 0.212 & 0.398 \\
\hline Total bilirubin (mg/dL) & 0.214 & 0.305 & -0.054 & 0.832 \\
\hline Alkaline phosphatase (U/L) & 0.230 & 0.268 & -0.238 & 0.341 \\
\hline Gamma-glutamyl transpeptidase (U/L) & -0.064 & 0.761 & 0.325 & 0.189 \\
\hline Alanine aminotransferase (U/L) & 0.498 & $0.011^{\star}$ & 0.240 & 0.338 \\
\hline Lactate dehydrogenase (U/L) & 0.157 & 0.452 & 0.156 & 0.536 \\
\hline Creatine kinase $(\mathrm{U} / \mathrm{L})$ & -0.227 & 0.275 & -0.382 & 0.118 \\
\hline Troponin (ng/L) & 0.142 & 0.498 & -0.236 & 0.345 \\
\hline C-reactive protein (mg/dL) & 0.011 & 0.958 & 0.176 & 0.485 \\
\hline Triglycerides (mg/dL) & 0.162 & 0.439 & 0.304 & 0.220 \\
\hline Cholesterol (mg/dL) & -0.219 & 0.292 & -0.255 & 0.308 \\
\hline
\end{tabular}

Statistical significance is from Pearson correlation test. ${ }^{\star} P<0.05,{ }^{* *} P<0.01$, and ${ }^{\star * *} P<0.001$. Bold lettering is shown when $P<0.05$. 
to exercise. Further research is needed to ascertain whether the strong intra-individual differences in the extent of GDF15 and FGF21 induction among runners can predict underlying adaptations and/or the risk for long-term damaging responses to the strenuous exercise associated with a marathon race.

\section{DATA AVAILABILITY STATEMENT}

The raw data supporting the conclusions of this article will be made available by the authors, without undue reservation.

\section{ETHICS STATEMENT}

The studies involving human participants were reviewed and approved by Comitè Ėtic de l'Hospital Universitari Germans Trias i Pujol (ICOR-2017- 04, REF.CEI, PI-17-037). The patients/participants provided their written informed consent to participate in this study.

\section{AUTHOR CONTRIBUTIONS}

ER, AB-G, and FV designed the study. DS-I and RC obtained the samples. LC, JV, and LN performed the analytical

\section{REFERENCES}

Adela, R., and Banerjee, S. K. (2015). GDF-15 as a target and biomarker for diabetes and cardiovascular diseases: a translational prospective. J. Diabetes Res. 2015:490842.

Artz, A., Butz, S., and Vestweber, D. (2016). GDF-15 inhibits integrin activation and mouse neutrophil recruitment through the ALK-5/TGF- $\beta$ RII heterodimer. Blood 128, 529-541. doi: 10.1182/blood-2016-01-696617

Bird, S. R., Linden, M., and Hawley, J. A. (2014). Acute changes to biomarkers as a consequence of prolonged strenuous running. Ann. Clin. Biochem. 51, 137-150. doi: 10.1177/0004563213492147

Bootcov, M. R., Bauskin, A. R., Valenzuela, S. M., Moore, A. G., Bansal, M., He, X. Y., et al. (1997). MIC-1, a novel macrophage inhibitory cytokine, is a divergent member of the TGF-beta superfamily. Proc. Natl. Acad. Sci. U.S.A. 94, 11514-11519. doi: 10.1073/pnas.94.21.11514

Conte, M., Martucci, M., Mosconi, G., Chiariello, A., Cappuccilli, M., Totti, V., et al. (2020). GDF15 plasma level is inversely associated with level of physical activity and correlates with markers of inflammation and muscle weakness. Front. Immunol. 11:915. doi: 10.3389/fimmu.2020.00915

Cuevas-Ramos, D., Almeda-Valdés, P., Meza-Arana, C. E., Brito-Córdova, G., Gómez-Pérez, F. J., Mehta, R., et al. (2012). Exercise increases serum fibroblast growth factor 21 (FGF21) levels. PLoS One 7:e38022. doi: 10.1371/journal.pone. 0038022

Emmerson, P. J., Wang, F., Du, Y., Liu, Q., Pickard, R. T., Gonciarz, M. D., et al. (2017). The metabolic effects of GDF15 are mediated by the orphan receptor GFRAL. Nat. Med. 23, 1215-1219. doi: 10.1038/nm.4393

Fudim, M., Kelly, J. P., Jones, A. D., AbouEzzeddine, O. F., Ambrosy, A. P., Greene, S. J., et al. (2020). Are existing and emerging biomarkers associated with cardiorespiratory fitness in patients with chronic heart failure? Am. Heart J. 220, 97-107. doi: 10.1016/j.ahj.2019.11.006

Fujita, Y., Ito, M., Kojima, T., Yatsuga, S., Koga, Y., and Tanaka, M. (2015). GDF15 is a novel biomarker to evaluate efficacy of pyruvate therapy for mitochondrial diseases. Mitochondrion 20, 34-42. doi: 10.1016/j.mito.2014.10.006

Galliera, E., Lombardi, G., Marazzi, M. G., Grasso, D., Vianello, E., Pozzoni, R., et al. (2014). Acute exercise in elite rugby players increases the circulating procedures. ER and FV wrote the manuscript. All authors discussed the data.

\section{FUNDING}

This study has been supported by grants SAF2017-85722-R, Ministerio de Ciencia, Innovación y Universidades, Spain; and FIS17/01455, Instituto de Salud Carlos III, Spain.

\section{ACKNOWLEDGMENTS}

The authors would like to thank the Barcelona marathon organization for their cooperation and the marathon runners for their participation.

\section{SUPPLEMENTARY MATERIAL}

The Supplementary Material for this article can be found online at: https://www.frontiersin.org/articles/10.3389/fphys. 2020.550102/full\#supplementary-material

level of the cardiovascular biomarker GDF-15. Scand. J. Clin. Lab. Invest. 74, 492-499. doi: 10.3109/00365513.2014.905697

Hagström, E., Held, C., Stewart, R. A., Aylward, P. E., Budaj, A., Cannon, C. P., et al. (2017). Growth Differentiation factor 15 predicts all-cause morbidity and mortality in stable coronary heart disease. Clin. Chem. 63, 325-333. doi: 10.1373/clinchem.2016.260570

Hansen, J. S., Clemmesen, J. O., Secher, N. H., Hoene, M., Drescher, A., Weigert, C., et al. (2015). Glucagon-toinsulin ratio is pivotal for splanchnic regulation of FGF-21 in humans. Mol. Metab. 4, 551-560. doi: 10.1016/j.molmet.2015.06.001 Hansen, J. S., Pedersen, B. K., Xu, G., Lehmann, R., Weigert, C., and Plomgaard, P. (2016). Exercise-induced secretion of FGF21 and follistatin are blocked by pancreatic clamp and impaired in type 2 diabetes. J. Clin. Endocrinol. Metab. 101, 2816-2825. doi: 10.1210/jc.2016-1681

Hsu, J. Y., Crawley, S., Chen, M., Ayupova, D. A., Lindhout, D. A., Higbee, J., et al. (2017). Non-homeostatic body weight regulation through a brainstemrestricted receptor for GDF15. GFRAL is the receptor for GDF15 and is required for the anti-obesity effects of the ligand. Nature 550, 255-259. doi: 10.1038 /nature24042

Kaleta-Duss, A. M., Lewicka-Potocka, Z., Dạrowska-Kugacka, A., Raczak, G., and Lewicka, E. (2020). Myocardial injury and overload among amateur marathoners as indicated by changes in concentrations of cardiovascular biomarkers. Int. J. Environ. Res. Public Health 17:6191. doi: 10.3390/ ijerph17176191

Kim, K. H., Kim, S. H., Min, Y. K., Yang, H. M., Lee, J. B., and Lee, M. S. (2013). Acute exercise induces FGF21 expression in mice and in healthy humans. PLoS One 8:e63517. doi: 10.1371/journal.pone.0063517

Kleinert, M., Clemmensen, C., Sjøberg, K. A., Carl, C. S., Jeppesen, J. F., Wojtaszewski, J. F. P., et al. (2018). Exercise increases circulating GDF15 in humans. Mol. Metab. 9, 187-191. doi: 10.1016/j.molmet.2017.12.016

Kobayashi, Y., Takeuchi, T., Hosoi, T., Yoshizaki, H., and Loeppky, J. A. (2005). Effect of a marathon run on serum lipoproteins, creatine kinase, and lactate dehydrogenase in recreational runners. Res. Q. Exerc. Sport 76, 450-455. doi: 10.1080/02701367.2005.10599318

Kratz, A., Lewandrowski, K. B., Siegel, A. J., Chun, K. Y., Flood, J. G., Van Cott, E. M., et al. (2002). Effect of marathon running on hematologic and biochemical 
laboratory parameters, including cardiac markers. Am. J. Clin. Pathol. 118, 856-863. doi: 10.1309/14ty-2tdj-1x0y-1v6v

Laurens, C., Parmar, A., Murphy, E., Carper, D., Lair, B., Maes, P., et al. (2020). Growth and differentiation factor 15 is secreted by skeletal muscle during exercise and promotes lipolysis in humans. JCI Insight. 5:e131870.

Lehtonen, J. M., Forsström, S., Bottani, E., Viscomi, C., Baris, O. R., Isoniemi, H., et al. (2016). FGF21 is a biomarker for mitochondrial translation and mtDNA maintenance disorders. Neurology 87, 2290-2299. doi: 10.1212/wnl. 0000000000003374

Montero, R., Yubero, D., Villarroya, J., Henares, D., Jou, C., Rodríguez, M. A., et al. (2016). GDF-15 is elevated in children with mitochondrial diseases and is induced by mitochondrial dysfunction. PLoS One 11:e0148709. doi: 10.1371/ journal.pone.0148709

Morville, T., Sahl, R. E., Trammell, S. A., Svenningsen, J. S., Gillum, M. P., Helge, J. W., et al. (2018). Divergent effects of resistance and endurance exercise on plasma bile acids, FGF19, and FGF21 in humans. JCI Insight. 3:e122737.

Mullican, S. E., Lin-Schmidt, X., Chin, C. N., Chavez, J. A., Furman, J. L., Armstrong, A. A., et al. (2017). GFRAL is the receptor for GDF15 and the ligand promotes weight loss in mice and nonhuman primates. Nat. Med. 23, 1150-1157. doi: 10.1038/nm.4392

Poulsen, N. S., Madsen, K. L., Hornsyld, T. M., Eisum, A. V., Fornander, F., Buch, A. E., et al. (2020). Growth and differentiation factor 15 as a biomarker for mitochondrial myopathy. Mitochondion 50, 35-41. doi: 10.1016/j.mito.2019.10. 005

Reid, S. A., Speedy, D. B., Thompson, J. M., Noakes, T. D., Mulligan, G., Page, T., et al. (2004). Study of hematological and biochemical parameters in runners completing a standard marathon. Clin. J. Sport Med. 14, 344-353. doi: 10.1097/ 00042752-200411000-00004

Ribas, F., Villarroya, J., Hondares, E., Giralt, M., and Villarroya, F. (2014). FGF21 expression and release in muscle cells: involvement of MyoD and regulation by mitochondria-driven signalling. Biochem. J. 463, 191-199. doi: 10.1042/ bj20140403

Sanchis-Gomar, F., Bonaguri, C., Aloe, R., Pareja-Galeano, H., Martinez-Bello, V., Gomez-Cabrera, M. C., et al. (2013). Effects of acute exercise and xanthine oxidase inhibition on novel cardiovascular biomarkers. Transl. Res. 162, 102109. doi: $10.1016 /$ j.trsl.2013.02.006

Sargeant, J. A., Aithal, G. P., Takamura, T., Misu, H., Takayama, H., Douglas, J. A., et al. (2018). The influence of adiposity and acute exercise on circulating hepatokines in normal-weight and overweight/obese men. Appl. Physiol. Nutr. Metab. 43, 482-490. doi: 10.1139/apnm-2017-0639

Slusher, A. L., Whitehurst, M., Zoeller, R. F., Mock, J. T., Maharaj, M., and Huang, C. J. (2015). Attenuated fibroblast growth factor 21 response to acute aerobic exercise in obese individuals. Nutr. Metab. Cardiovasc. Dis. 25, 839-845. doi: 10.1016/j.numecd.2015.06.002

Stahrenberg, R., Edelmann, F., Mende, M., Kockskämper, A., Düngen, H. D., Lüers, C., et al. (2010). The novel biomarker growth differentiation factor 15 in heart failure with normal ejection fraction. Eur. J. Heart Fail. 12, 1309-1316. doi: 10.1093/eurjhf/hfq151

Suomalainen, A., Elo, J. M., Pietiläinen, K. H., Hakonen, A. H., Sevastianova, K., Korpela, M., et al. (2011). FGF-21 as a biomarker for muscle-manifesting mitochondrial respiratory chain deficiencies: a diagnostic study. Lancet Neurol. 10, 806-818. doi: 10.1016/s1474-4422(11)70155-7

Tanimura, Y., Aoi, W., Takanami, Y., Kawai, Y., Mizushima, K., Naito, Y., et al. (2016). Acute exercise increases fibroblast growth factor 21 in metabolic organs and circulation. Physiol. Rep. 4:e12828. doi: 10.14814/phy2. 12828

Tchou, I., Margeli, A., Tsironi, M., Skenderi, K., Barnet, M., Kanaka-Gantenbein, C., et al. (2009). Growth-differentiation factor-15, endoglin and N-terminal probrain natriuretic peptide induction in athletes participating in an ultramarathon foot race. Biomarkers 14, 418-422. doi: 10.1080/13547500903062976

Tsai, V. W. W., Husaini, Y., Sainsbury, A., Brown, D. A., and Breit, S. N. (2018). The MIC-1/GDF15-GFRAL pathway in energy homeostasis: implications for obesity, cachexia, and other associated diseases. Cell Metab. 28, 353-368. doi: 10.1016/j.cmet.2018.07.018

Weigert, C., Hoene, M., and Plomgaard, P. (2019). Hepatokines-a novel group of exercise factors. Pflugers. Arch. 471, 383-396. doi: 10.1007/s00424-018-2216-y

Yang, L., Chang, C. C., Sun, Z., Madsen, D., Zhu, H., Padkjær, S. B., et al. (2017). GFRAL is the receptor for GDF15 and is required for the anti-obesity effects of the ligand. Nat. Med. 23, 1158-1166. doi: 10.1038/nm.4394

Yatsuga, S., Fujita, Y., Ishii, A., Fukumoto, Y., Arahata, H., Kakuma, T., et al. (2015). Growth differentiation factor 15 as a useful biomarker for mitochondrial disorders. Ann. Neurol. 78, 814-823. doi: 10.1002/ana.24506

Conflict of Interest: The authors declare that the research was conducted in the absence of any commercial or financial relationships that could be construed as a potential conflict of interest.

Copyright (C) 2020 Campderrós, Sánchez-Infantes, Villarroya, Nescolarde, BayèsGenis, Cereijo, Roca and Villarroya. This is an open-access article distributed under the terms of the Creative Commons Attribution License (CC BY). The use, distribution or reproduction in other forums is permitted, provided the original author(s) and the copyright owner(s) are credited and that the original publication in this journal is cited, in accordance with accepted academic practice. No use, distribution or reproduction is permitted which does not comply with these terms. 